\title{
Acompañamiento espiritual virtual: alcances y límites*
}

\author{
Edgar Enrique Velásquez Camelo, SDB ${ }^{\mathrm{a}}$ \\ Sociedad de San Francisco de Sales \\ http:/ / orcid.org/0000-0003-1359-3848
}

RECIBIDO: 08-08-19. APROBADO: 27-11-2019

Resumen: La virtualización de las dinámicas de interacción social del mundo actual ha constituido diversas formas de relación entre las personas que hacen parte de la sociedad red. El diálogo, el compartir espontáneo, una conversación tienen su escenario de acción en los procesos de socialización que se llevan a cabo desde las plataformas digitales de comunicación. También, las dinámicas en torno de la experiencia de fe se han virtualizado y con ella las dimensiones de la pastoral de la Iglesia (kerigmática, comunitaria, diaconía, litúrgica y la catequesis); esto nos lleva a pensar la posibilidad del acompañamiento espiritual virtual como una acción pastoral de la Iglesia dirigida a las personas que, profesando la fe en Cristo Jesús, permanecen en la red. El objetivo de este artículo es presentar una reflexión que dé cuenta de los alcances y límites del acompañamiento espiritual virtual en la búsqueda personal del sentido de la existencia y la maduración de la vida de fe; así mismo, establecer las condiciones de posibilidad que llevan a plantear la viabilidad de direccionar procesos de acompañamiento espiritual por medio de las redes sociales que facilitan el diálogo, la cultura del encuentro y la participación activa de las personas en la sociedad red. Los alcances de dicha modalidad de acompańamiento están determinados por el grado de vinculación vital y referencial que establecen las personas por medio de los dispositivos electrónicos, y los límites están demarcados por la capacidad de tomar distancia del mundo virtual, es decir, el saber integrar en la existencia, de forma armónica, la representación virtual-real como actual-real del mundo. Todo esto en orden a enriquecer las prácticas eclesiales en la cultura de la virtualidad real y, además, legitimar la reflexión ciberteológica que tiene un estatuto epistemológico genuino que emerge en la relación integral entre fe, razón y vida.

Palabras Clave: Ciberteología; virtualización; acompañamiento espiritual; redes sociales; permanecer; evangelización; comunicación y experiencia de fe.

*Artículo de reflexión.

a Autor de correspondencia. Correo electrónico: evelasquez@sdbcob.org 


\section{Virtual Spiritual Accompaniment: Scope and Limits}

AвSTRACT: The virtualization of social interaction dynamics in today's world has constituted various forms of relationship between people who are part of the network society. Dialogue, spontaneous sharing, a conversation all have their stage of action in the socialization processes that takes place from the digital communication platforms. Also, the dynamics around the experience of faith have been virtualized and with it the dimensions of the pastoral of the Church (kerygmatic, community, diakonia, liturgical and catechesis); this leads us to think of the possibility of virtual spiritual accompaniment as a pastoral action of the Church directed to those who, professing faith in Jesus Christ, remain in the network. The aim of this article is to present a reflection that gives an account of the scope and limits of virtual spiritual accompaniment in the personal search for the meaning of existence and the maturation of the life of faith; also, to establish the conditions of possibility that lead to the feasibility of directing processes of spiritual accompaniment through social networks that facilitate dialogue, the culture of encounter and the active participation of people in the network society. The scope of this form of accompaniment is determined by the degree of vital and referential connection established by people through electronic devices, and the limits are demarcated by the ability to distance oneself from the virtual world, that is, the knowledge to integrate in the existence, in a harmonic way, the virtual-real representation as actual-real of the world. All this in order to enrich ecclesial practices in the culture of real virtuality and, in addition, to legitimize the cybertheological reflection that has a genuine epistemological statute that emerges in the integral relationship between faith, reason and life.

KeY Words: Cybertheology; Virtualization; Spiritual accompaniment; Social networks; To stay; Evangelization; Communication and faith experience.

\section{CÓMO CITAR:}

Velásquez Camelo, Edgar E. “Acompañamiento espiritual virtual: alcances y límites”. Theologica Xaveriana (2021): 1-26. https://doi.org/10.11144/javeriana.tx71.aeval

\section{ReCONOCIMIENTO}

El quehacer teológico académico y pastoral adelantado en la Pontificia Universidad Javeriana, en la Facultad de Teología, me ha llevado a reconocer, como estudiante, la importancia de estar en constante actualización, para no perder de vista los desafíos que nos presenta el mundo de hoy. De ahí el interés por publicar este artículo, que es producto de la reflexión acerca de la relación entre fe, cultura y vida. Agradezco a quiénes acompañaron en el proceso de investigación: a la profesora Edith González Bernal, a los profesores David Lara Corredor y Daniel de Jesús Garavito, al padre Hernán Darío Cardona y a la religiosa Sandra Milena Acosta. 


\section{Introducción}

El acompańamiento espiritual virtual no es un tema nuevo. En el primer siglo del cristianismo, el Apóstol de los gentiles ya usaba medios virtuales de acompañamiento a las comunidades por medio de cartas: hacía presencia virtual en la escritura ${ }^{1}$. Por mencionar otro ejemplo, San Francisco de Sales también hizo acompañamiento por medio de cartas a personas particulares de diversa condición². Ahora bien, si la mayoría de las personas en la actualidad han virtualizado sus relaciones interpersonales en la red, tiene sentido pensar en las estrategias pastorales de la presencia continua de la Iglesia en el mundo virtual.

Por eso nos preguntamos: ¿En qué sentido las redes sociales son una oportunidad para el acompañamiento espiritual virtual en el mundo actual? ¿Es posible un acompañamiento espiritual virtual? De ser así, ¿cuáles son sus alcances y límites? En este artículo se defiende la hipótesis de que en la actualidad es posible, urgente y necesario el cultivo de la vida espiritual; dado que hay un creciente interés por permanecer conectado en la red, se hace necesario el cultivo de la vida espiritual por medio del acompañamiento virtual, que permita a las personas descubrir caminos auténticos de realización humana que respondan a las necesidades concretas de su existencia.

Para desarrollar esta hipótesis proponemos el siguiente camino: en primer lugar, dar cuenta del estado actual de la ciberteología; en segundo lugar, presentar la relación concomitante y dialógica entre fe, cultura y vida; en tercer lugar, desarrollar los alcances y límites del acompañamiento espiritual virtual, de tal forma que se pueda responder a los retos de los que permanecen en la red.

\section{La Iglesia y el mundo virtual: aproximación}

La virtualización del mundo social ha fundado diversas formas de crear y recrear el mundo institucional, a tal punto, que los diferentes subsistemas sociales del mundo real-actual tienen su representación en Internet ${ }^{3}$. Cada grupo social, partido político, comunidad, grupo de contactos unidos por múltiples propósitos y gustos, la institu-

\footnotetext{
${ }^{1}$ Herranz hace un estudio exhaustivo acerca de Pablo a partir de sus cartas. Uno de los rasgos que sale a la vista es la finalidad de dichas cartas, es decir, el modo como él hacía presencia en las comunidades por medio de la escritura. Si, con Pierre Levy, podemos afirmar que la escritura es una expresión de la virtualización, entonces se puede afirmar que San Pablo hacía presencia virtual por medio de la escritura (ver a Herranz, San Pablo en sus cartas).

${ }^{2}$ Bustamante, La práctica de la dirección espiritual en la vida y enseñanzas de San Francisco de Sales.

${ }^{3}$ Velásquez, La virtualización en el escenario contemporáneo de la educación.
} 
ción de cada estamento gubernamental y otras formas de asociación tienen su espacio en la red.

De igual forma, la Iglesia Católica está presente en el mundo virtual-real, tanto con la mera presentación de información de millones de datos como -sobre todo- para hacer posible que en el mundo virtual se lleven a cabo procesos de evangelización. Así lo hacía notar de forma profética el Concilio Vaticano II, con el decreto conciliar Inter mirifica, al exponer la siguiente necesidad:

Procure, de común acuerdo, todos los hijos de la Iglesia, que los instrumentos de comunicación social se utilicen eficazmente, sin la menor dilación y con el máximo empeño, en las más variadas formas apostolado, tal como lo exigen las realidades y las circunstancias de nuestro tiempo, adelantándose así a las malas iniciativas, especialmente en aquellas regiones en las que el progreso moral y religioso reclama una mayor atención. ${ }^{4}$

Si bien el decreto conciliar Inter mirifica (aprobado y publicado el 5 de diciembre de 1963)5 presentó las líneas generales acerca de la relación que debía tener la Iglesia con los medios de comunicación, sobre todo la evangelización por medio de la radio, la televisión, el cine, la prensa y libros, solo seis años después, en 1969, cuando se data el origen de la Internet como herramienta comunicativa militar denominada Arpanet $^{6}$. Nos parece pertinente hacer esta anotación porque actualmente Internet recoge las diversas formas de comunicación virtual, compone el entramado informático virtual de los medios de comunicación y es además el escenario digital donde la Iglesia puede -como afirma el Concilio- sin la menor dilación y con el máximo empeño, desarrollar las múltiples formas de apostolado posible para responder a las necesidades de las personas que hacen posible la instauración de la cultura de la virtualidad real'7.

Inter mirifica marcó un nuevo camino para la evangelización del mundo, abrió la puerta a múltiples formas de apostolado digital y fue el punto de partida de la riqueza magisterial sobre los medios de comunicación social como herramientas óptimas para las nuevas formas de comunicar al señor Jesús ${ }^{8}$. Posteriormente se publicó una instrucción pastoral, Communio et progressio, con el fin de profundizar los aspectos

\footnotetext{
${ }^{4}$ Concilio Vaticano II, "Decreto conciliar Inter mirifica sobre los medios de comunicación social” 13.

${ }^{5}$ Ibíd.

${ }^{6}$ Castells, La galaxia Internet. Reflexiones sobre Internet, empresa y sociedad, 23.

${ }^{7}$ Castells, La era de la información. La sociedad red, Vol. 1, 398. 443

${ }^{8}$ Guitián, "El Vaticano II y los medios de comunicación: a los cincuenta años de Inter mirifica".
} 
poco desarrollados por el Concilio, que resalta el papel de los medios de comunicación social como instrumentos de evangelización, de comunión y participación?.

De ahí que "se debe a Inter mirifica que la Iglesia se haya tomado en serio la tarea de profundizar doctrinalmente en la realidad de la comunicación social, y que en cada lugar se haya producido un despertar, o incluso el nacimiento, de la conciencia de la importancia de los medios de comunicación" ${ }^{10}$.

Luego de estos documentos fueron surgiendo otros que soportaron la reflexión sobre la importancia de los medios de comunicación social en la Iglesia, sobre las implicaciones éticas y morales, la comunión del mundo, la instauración de una nueva forma de configurar la realidad social y eclesial, entre otros asuntos ${ }^{11}$. Los medios de comunicación representan los nuevos areópagos de intercambio social y cultural que hacen cada vez más posible convertir al mundo en una "aldea global" de interacción ${ }^{12}$. Pablo VI, en Evangelii nuntiandi, afirmó acerca de los medios de la evangelización:

...la evidente importancia del contenido no debe hacer olvidar la importancia de los métodos y medios de la evangelización. Este problema de cómo evangelizar es siempre actual, porque las maneras de evangelizar cambian según las diversas circunstancias de tiempo, lugar, cultura; por eso plantean casi un desafío a nuestra capacidad de descubrir y adaptar. ${ }^{13}$

Los medios permiten comunicar la experiencia de fe. Aunque la tecnología ofrece formas variadas de comunicación, la forma privilegiada de anunciar a Cristo seguirá siendo el testimonio de vida, la liturgia de la vida, la catequesis, el encuentro personal, la vida sacramental y la piedad popular ${ }^{14}$. Si un cristiano, convencido de su $\mathrm{fe}$, es testimonio de vida ejemplar, vive una buena relación con Dios y las personas, y

9 Pontificia Comisión para los Medios de Comunicación Social, "Instrucción pastoral Communio et progressio sobre los medios de comunicación social (1971)".

${ }^{10}$ Ibíd. 636.

${ }^{11}$ Véanse los siguientes documentos del Pontificio Consejo para las Comunicaciones Sociales: "Pornografía y violencia en las comunicaciones sociales: una respuesta pastoral (1989)"; "Criterios de colaboración ecuménica e interreligiosa en las comunicaciones sociales (1989)"; "Instrucción pastoral Aetatis novae sobre las comunicaciones sociales en el vigésimo aniversario de Communio et progressio (1992)"; "Ética en la publicidad (1997)"; "Ética de las comunicaciones sociales (2000)"; "Ética en Internet (2002)"; "La Iglesia en Internet (2002)"; de Juan Pablo II, "Carta apostólica 'El rápido desarrollo' a los responsables de las comunicaciones sociales (2005)"; y los diversos mensajes desde Pablo VI hasta el papa Francisco en las Jornadas de Comunicación Social.

${ }^{12}$ McLuham, La aldea global. Transformaciones en la vida y los medios de comunicación mundiales en el siglo XXI.

${ }^{13}$ Pablo VI, "Exhortación apostólica Evangelii nuntiandi acerca de la evangelización en el mundo contemporáneo (1975)" 40.

${ }^{14}$ Ibíd. 41-48. 
utiliza todos los medios posibles para dar a conocer su experiencia de Dios, hace mucho bien a la comunidad eclesial que al conocer su vivencia de Dios se siente conminada a vivir de acuerdo con el Evangelio. En concreto, sobre los medios de comunicación Pablo VI advierte:

En nuestro siglo influenciado por los medios de comunicación social, el primer anuncio, la catequesis o el ulterior ahondamiento de la fe no pueden prescindir de esos medios, como hemos dicho antes. Puestos al servicio del Evangelio, ellos ofrecen la posibilidad de extender casi sin límites el campo de audición de la Palabra de Dios, haciendo llegar la Buena Nueva a millones de personas. La Iglesia se sentiría culpable ante Dios si no empleara esos poderosos medios, que la inteligencia humana perfecciona cada vez más. Con ellos la Iglesia "pregona sobre los terrados" el mensaje del que es depositaria. En ellos encuentra una versión moderna y eficaz del "púlpito". Gracias a ellos puede hablar a las masas. Sin embargo, el empleo de los medios de comunicación social en la evangelización supone casi un desafío: el mensaje evangélico deberá, sí, llegar, a través de ellos, a las muchedumbres, pero con capacidad para penetrar en las conciencias, para posarse en el corazón de cada hombre en particular, con todo lo que este tiene de singular y personal, y con capacidad para suscitar en favor suyo una adhesión y un compromiso verdaderamente personal. ${ }^{15}$

Aunque la comunicación por medio de la tecnología sea masificada, Pablo VI indica la necesidad que la evangelización sea personalizada, para suscitar en cada creyente un profundo deseo de adhesión a Cristo. Con esta advertencia, el santo Padre reconoce el potencial evangelizador de los medios de comunicación y la necesidad de personalizar la evangelización virtual, es decir, que sea, además de masificadora, personal.

La personalización del anuncio del Evangelio en el mundo virtual es posible ahora, por medio de las redes sociales de interacción y comunicación que permiten que el anuncio de Jesucristo sea más concreto y explícito en cada una de las plataformas comunicativas que ofrece Internet. Si bien los instrumentos tecnológicos son simplemente herramientas, no se puede desconocer que actualmente el medio-como señala McLuhan- es el mensaje ${ }^{16}$.

En sus mensajes recientes durante las jornadas mundiales de las Comunicaciones Sociales, el papa Francisco ha resaltado el papel preponderante de la tecnología en la creación de la cultura del encuentro ${ }^{17}$, la facilidad de la comunicación intercultural en

\footnotetext{
${ }^{15}$ Ibíd. 45.

${ }^{16}$ McLuhan, Comprender los medios de comunicación. Las extensiones del ser humano, 29

${ }^{17}$ Francisco, "Mensaje para la XLVIII Jornada Mundial de las Comunicaciones Sociales. 'Comunicación al servicio de una auténtica cultura del encuentro' (2014)".
} 
un mundo globalizado, la importancia de la comunicación entre la familia (es decir, la necesidad de crear un ambiente de comunión familiar positivo, lleno de palabras de amor, cariño, misericordia y comprensión ${ }^{18}$; t también ha resaltado la necesidad de que los medios de comunicación se expresen en el lenguaje de la misericordia que posibilite un encuentro fecundo en las redes ${ }^{19} \mathrm{y}$ de que las comunicaciones estén atravesadas por la esperanza y la confianza, y no por los mensajes noticiosos amarillistas que inundan los canales de televisión informativos ${ }^{20}$; igualmente ha enfatizado en la importancia que tienen las noticias veraces para hacer posible un entorno social en paz ${ }^{21}$.

Podemos destacar que los campos de reflexión sobre los medios de comunicación no han sido agotados, ni mucho menos se ha abordado la complejidad de las cuestiones relacionadas con la virtualización del mundo social, en temas como la identidad, la educación, la política red, el contenido multimedia, las redes sociales, la opinión pública, el cine, la radio, la televisión, entre otros.

El de 2014 fue el primero de los mensajes del papa Francisco para las sucesivas jornadas mundiales de las Comunicaciones Sociales; en él resaltó la importancia de la cultura del encuentro, señalando que las redes sociales son verdaderos escenarios de intercambio social que posibilitan, entre otras, la relación entre personas que han virtualizado su existencia por medio de perfiles identificatorios ${ }^{22}$ y que se han aglomerado en diversos grupos de interés con criterios de conformación de acuerdo con los gustos o afinidades encontrados en la red.

Si la comunicación constituye la forma como el ser humano se ha hecho lenguaje, la máquina, como un interceptor lógico, ha hecho que el lenguaje humano se vuelva parte constitutiva del lenguaje de máquina que configura la red $^{23}$. Decir el mundo por medio de la máquina es virtualizar las múltiples formas de la socialización humana. La máquina -laptops y celulares principalmente- son las herramientas que han creado en la sociedad red, variadas formas de comunicación y participación en el mundo digitalmente posible y accesible en la pantalla.

\footnotetext{
${ }^{18}$ Francisco, "Mensaje para la XLIX Jornada Mundial de las Comunicaciones Sociales. 'Comunicar la familia: ambiente privilegiado del encuentro en la gratuidad del amor' (2015)”.

${ }^{19}$ Francisco, "Mensaje para la 50 Jornada Mundial de las Comunicaciones Sociales. 'Comunicación y misericordia: un encuentro fecundo' (2016)".

${ }^{20}$ Francisco, "Mensaje para la 51 Jornada Mundial de las Comunicaciones Sociales. 'No temas, que yo estoy contigo' (Is 43,5). Comunicar esperanza y confianza en nuestros tiempos' (2017)”.

${ }^{21}$ Francisco, "Mensaje para la 52 Jornada Mundial de las Comunicaciones Sociales. 'La verdad os hará libres (Jn 8,32). Fake news y periodismo de paz' (2018)”.

${ }^{22}$ Velásquez, La virtualización en el escenario contemporáneo de la educación.

${ }^{23}$ Velásquez, "La virtualización. Aproximación desde Manuel Castells y Jean Baudrillard”.
} 
Si Internet ha permitido la comunicación abierta y a distancia es posible pensar en las muchas formas de apostolado que se pueden realizar, desde la publicación de materiales multimedia de carácter religioso, en las distintas plataformas virtuales de interacción social, también llamadas redes sociales, hasta las conversaciones que versan sobre el sentido de la vida, las realidades últimas, la vida espiritual y, por qué no decirlo, sobre la relación con Dios.

En este sentido, la ciberteología -reflexión que versa sobre la revelación que acontece en la acción humana por medio de las plataformas virtuales de interacción social- permite reconocer la influencia de los medios de comunicación social en la experiencia de Dios y las posibilidades inagotables de testimoniar la propia experiencia de fe en las redes sociales ${ }^{24}$. La persona, al hacer experiencia de Dios, al encontrarse con el señor Jesús, sentir su amor y misericordia, siente el deseo de comunicar al mundo lo vivido en su corazón.

Análogamente, al sentirse vacías, sin sentido, sin motivaciones, sin aspiraciones profundas en la vida, las personas desean dar a conocer al mundo las múltiples problemáticas que atraviesan su existencia, con el deseo de resolver la crisis de alguna forma. Así, en la base de las dos experiencias, la de Dios y la del sinsentido, está la comunicación. Si en la actualidad las redes virtuales de interacción son escenarios privilegiados de comunicación humana, tiene sentido pensar que en ellas se comparte la complejidad existencial de la vida de las personas: sus logros, retrocesos, afinidades, gustos, convicciones, su realidad íntima o superficial, sus aspiraciones y proyecciones, su relación con Dios y su búsqueda del sentido de la vida.

Las redes sociales son espacios virtuales de comunicación que facilitan los procesos de interacción social con el mundo, tanto virtual como actual; es decir, su propósito es afectar las relaciones en las mismas redes y las que se viven en la cotidianidad. En estos espacios se comparte variedad de contenidos que tienen que ver con la realidad personal, con las tendencias del momento -políticas, sociales, culturales-, o con algún gusto individual o colectivo. Las comunidades virtuales creadas en las redes están llamadas a ser verdaderos espacios de socialización humana, con el fin de comunicar la vida actual virtualizada en el dispositivo.

\section{Diálogo entre fe, cultura y vida}

Los fundamentos epistemológicos de la ciberteología parten de la relación entre fe, cultura y vida. No hay que desconocer que la vida de las personas y de los creyentes, hoy

\footnotetext{
${ }^{24}$ Arboleda, “Evangelizar la cibercultura. Los retos de la ciberteología”, 163-181.
} 
en día, se desenvuelve en relación continua, directa y permanente con la tecnología ${ }^{25}$. De ahí se desprende la razón fundamental que legitima la reflexión ciberteológica, sobre todo porque los aspectos esenciales de la vida del ser humano acontecen en las dinámicas intersubjetivas que suceden en la red ${ }^{26}$.

Asuntos como la comunicación, el intercambio simbólico cultural, la configuración del sistema de creencias que orienta la vida cotidiana de las personas, la experiencia de fe, inclusive nuevas formas de brindar sentido de la vida por medio de la dirección espiritual virtual son aspectos esenciales que forman parte de la reflexión que surge en la relación entre la fe y el mundo digital.

Los presupuestos que hay de fondo para el desarrollo de esta propuesta son los siguientes:

1. La vida de las personas -en las sociedades desarrolladas o en vías de desarrollose desenvuelve de manera predominante en relación con la tecnología ${ }^{27}$. Los nativos digitales ${ }^{28}$ configuran un ámbito específico de la reflexión teológica, al establecer, en su relación con el mundo digital, la interacción entre el conjunto de creencias que configura el pensamiento cotidiano no especializado sobre la vida desde la red y las verdades que se heredan de la tradición enriquecen la comprensión del ser humano en la sociedad red.

2. La cultura de la virtualidad real define los mecanismos que construyen la sociedad red caracterizado por ser "espacios de flujos" en continua movilidad y transformación ${ }^{29}$.

3. La fe es un elemento central que comprende al ser humano en relación directa con la experiencia religiosa. Hoy en día la fe se expresa (aunque no solamente) por medio de las tecnologías de la información y la comunicación; en otras palabras, las tecnologías son capaces de crear múltiples entornos simbólicos representativos para compartir la fe.

Pensar el discurso teológico en relación directa con las tecnologías de la información y la comunicación es una tarea preponderante, sobre todo, porque la vida del ser humano en la actualidad se desenvuelve de manera predominante en la red. En otras palabras, el escenario virtual de interacción social se ha convertido en el ámbito en

\footnotetext{
${ }^{25}$ Velásquez, La virtualización en el escenario contemporáneo de la educación.

${ }^{26}$ Spadaro, Ciberteología. Pensar el cristianismo en tiempos de la red.

${ }^{27}$ Velásquez, "La dimensión temporal del Dasein en la era virtual: para una hermenéutica existencial del tiempo".

${ }^{28}$ Spadaro, Ciberteología. Pensar el cristianismo en tiempos de red.

${ }^{29}$ Castells, La era de la información. La sociedad red. Vol. 1.
} 
donde se puede compartir la fe, conocer diferentes cosmovisiones religiosas, ancladas a un contexto cultural específico, y propiciar experiencias significativas que promuevan al ser humano a la transcendencia.

Si bien la tecnología es una oportunidad en los múltiples campos de socialización humana, también representa una amenaza, porque está en capacidad de dirigir la opinión pública, conducir a las personas a la aceptación casi ciega de un sistema de valores - muchas veces contradictorios entre sí-, y exaltar el reino de la habladuría y la apariencia $^{30}$. Esto no nos puede desanimar en la empresa de querer exaltar los beneficios de la red, e ignorar su potencial transformador del mundo social en la consecución de un modo quizás más humano ${ }^{31}$.

La apertura al mundo moderno por parte de la Iglesia con el Concilio Vaticano II se convirtió en el punto de partida para realizar una teología que atiende a los signos de los tiempos ${ }^{32}$. Aquí resulta iluminador el diálogo entre fe, cultura y vida, toda vez que en el dinamismo de estos elementos surgen las bases fundamentales de la epistemología de la ciberteología. El elemento integrador entre fe y cultura es la vida del ser humano; quien vive la fe y la expresa con los elementos de la cultura es la persona del creyente.

Además, si no se puede desconocer la cultura en correlación directa con la fe, entonces, tiene sentido pensar que la sociedad red instaurada por la proliferación de las tecnologías que virtualizan la realidad social hace parte del dinamismo vital que configura una nueva hermenéutica existencial del tiempo ${ }^{33}$. Ahora la tecnología no es solo una herramienta externa, sino hace parte integral de la vida de las personas.

No hay que olvidar que las herramientas, como conjunto de objetos útiles, hacen parte momentánea de la vida de la persona determinados por un uso específico ${ }^{34}$. Estos objetos son, al modo de la jarra de Heidegger, en cuanto cosa determinada por su uso, para lo cual fue hecha como jarra ${ }^{35}$. Sin embargo, jes posible hacer esta misma lectura con las tecnologías de la información y la comunicación? La tecnología hace parte del conjunto de herramientas que cada logran afectar vez más la vida de las personas. Por esto no son solo herramientas; por ejemplo, un destornillador hace parte del

\footnotetext{
${ }^{30}$ Baudrillard, La ilusión vital; y a Sartori, Homo videns: sociedad teledirigida.

${ }^{31}$ Vattimo, "La sociedad transparente".

${ }^{32}$ Concilio Vaticano II, Concilio Vaticano II. "Constitución pastoral Gaudium et spes sobre la Iglesia en el mundo actual (1965)".

${ }^{33}$ Velásquez, "La dimensión temporal del Dasein en la era virtual: para una hermenéutica existencial del tiempo".

${ }^{34}$ Velásquez, "La sociedad de consumo en La Caverna de Saramago."

${ }^{35}$ Heidegger, La pregunta por la cosa la doctrina kantiana de los principios trascendentales.
} 
conjunto de cosas que cumplen una función determinada al modo de la jarra; pero un Smartphone hace parte del sistema de objetos ${ }^{36}$ que constituyen el universo simbólico virtual que ya no son simplemente herramientas, sino los elementos que configuran la cultura de la virtualidad real ${ }^{37}$.

¿Cómo afecta esto la fe de la persona que ha virtualizado su existencia? ¿Qué papel cumplen las tecnologías en la configuración de la fe y la identidad del creyente? ¿Cuáles son las características de la cultura de la virtualidad real que afectan de forma preponderante la experiencia de fe? ¿En qué forma la existencia virtual de la persona se convierte en un nuevo escenario donde es posible hacer teología? ¿Qué desafíos teológicos se desprenden en la relación entre la vida, la sociedad red que afecta en la búsqueda por el sentido de la vida?

Estas cuestiones tienen de fondo la relación entre fe, cultura y vida. La tecnología ocupa un lugar relevante que determina el entorno cultural. Según Spadaro:

...la red no es en absoluto un simple "instrumento" de comunicación que se puede usar o no, sino que se ha convertido en un espacio, un "ambiente" cultural, que determina un estilo de pensamiento y que crea nuevos territorios y nuevas formas de educación, contribuyendo a definir también una nueva manera de estimular la inteligencia y estrechar las relaciones, un modo incluso de habitar el mundo y de organizarlo. ${ }^{38}$

El carácter instrumental de la tecnología es su naturaleza más específica; sin embargo, las tecnologías de la información y la comunicación se han convertido en la base fundamental de la conformación del orden social generalizado ${ }^{39}$. No podemos desconocer que las tecnologías son parte integral del mundo actual y que, a través de ellas, se pueden llevar a cabo procesos de transformación, lo cual implica también, a como dé lugar, un cambio de mentalidad ${ }^{40}$.

Si bien podemos tener cierta suspicacia -como afirma Baudrillard- de la realidad virtual, porque en ella se imprime un carácter aparente e ilusorio del mundo ${ }^{41}$, esto no quiere decir dejar de lado el potencial comunicador y transformador que pueden suscitar los entornos culturales virtuales. La Iglesia del siglo XXI no puede desconocer que el ser humano de esta época es virtual y actual, que vive en el mundo

\footnotetext{
${ }^{36}$ Baudrillard, El sistema de objetos.

${ }^{37}$ Sínodo de Obispos, "XV Asamblea General Ordinaria "Los jóvenes, la fe y el discernimiento vocacional. Documento final (2018)".

${ }^{38}$ Spadaro, Ciberteología. Pensar el cristianismo en tiempos de la red, 23.

${ }^{39}$ Vattimo, "La sociedad transparente".

${ }^{40}$ Velásquez, La virtualización en el escenario contemporáneo de la educación.

${ }^{41}$ Baudrillard, La ilusión vital.
} 
de la máquina y, a la vez, en un entorno real de interacción social. Esta característica no hace más importante un aspecto frente al otro; antes, por el contrario, se puede afirmar que el mundo real de interacción social y el mundo virtual de comunicación cultural son las dos caras de la misma moneda: la complejidad existencial virtual del ser humano ${ }^{42}$.

Si bien -según Spadaro- la ciberteología se ha abordado en los campos de la teología pastoral, la teología aplicada, los aspectos trinitarios de la comunicación, el uso de los medios de comunicación para la evangelización, la creación de contenidos, la comunicación y la teología, en la actualidad falta mayor realce al carácter teológico de la tecnología como posibilidad misma de reconocerla, incluso, como un lugar de la revelación ${ }^{43}$.

Aquí no hay que pensar en cosas muy elevadas o abstractas, sino, más bien, en la realidad concreta del ser humano virtual. ¿Cuáles serían esas expresiones propias que nos ayudan a entender la tecnología como ámbito privilegiado de la revelación? Un modo concreto que hace patente la revelación de Dios en Internet es el acompañamiento espiritual virtual. ¿En qué sentido? Así como Internet configura, según Manuel Castells, un entorno digital de interacción social, y quienes hacemos parte de dicha interacción somos todos los que hemos virtualizado las relaciones sociales en la red, es posible generar procesos de comunicación que vinculen estrechamente el mundo virtual-real y actual real ${ }^{44}$.

Estos procesos de comunicación hacen posible, de igual modo, el testimonio, acompañamiento del nombre cristiano. Somos cristianos en la red como en el mundo actual real; dicho de otra manera, la identidad del creyente se puede comunicar con mucha fuerza, tanto en el mundo actual como en los entornos culturales virtuales de la red. Si todo cristiano tiene la responsabilidad, en virtud del bautismo, de ser testigo de Cristo Jesús en el mundo, no tiene sentido que solo se haga dicha comunicación en el mundo actual real, cuando la mayor parte del tiempo (por no decir siempre) está virtualmente presente y conectado en el mundo digital.

\footnotetext{
${ }^{42}$ Velásquez, "La dimensión temporal del Dasein en la era virtual: para una hermenéutica existencial del tiempo".

${ }^{43}$ Spadaro, Ciberteología. Pensar el cristianismo en tiempos de la red, 36.

${ }^{44}$ Castells, La galaxia Internet. Reflexiones sobre Internet, empresa y sociedad.
} 


\section{Oportunidades de la ciberteología: virtualización de la fe en la cultura de la virtualidad real}

El mensaje del papa Francisco en la Jornada Mundial de las Comunicaciones Sociales, en 2014, nos ofreció ideas contundentes, de alto contenido teológico, acerca de la tecnología, en particular de Internet:

En este mundo, los medios de comunicación pueden ayudar a que nos sintamos más cercanos los unos de los otros, a que percibamos un renovado sentido de unidad de la familia humana que nos impulse a la solidaridad y al compromiso serio por una vida más digna para todos. [...] La cultura del encuentro requiere que estemos dispuestos no solo a dar, sino también a recibir de los otros. Los medios de comunicación pueden ayudarnos en esta tarea, especialmente hoy, cuando las redes de la comunicación humana han alcanzado niveles de desarrollo inauditos. En particular, Internet puede ofrecer mayores posibilidades de encuentro y de solidaridad entre todos; y esto es algo bueno, es un don de Dios. ${ }^{45}$

Internet es un espacio teologal donde acontece, de forma contingente, la revelación de Dios. La contingencia revelatoria de Dios se debe a que acontece en la amalgama informacional de la red mundial, que no siempre transmite a Dios de forma explícita ${ }^{46}$. De la misma manera como la palabra humana es contingente, los medios comunicaciones virtuales, aunados en Internet, son limitados. No resulta fácil identificar los elementos que constituyen la revelación de Dios en las plataformas virtuales de interacción social, sobre todo porque allí acontece lo que está al servicio del capitalismo informacional ${ }^{47}$.

Dios se hace virtual para darse a conocer al ser humano que ha virtualizado su existencia, para comunicarle su mensaje de salvación, es decir, liberarlo del egoísmo asfixiante de la red. La virtualidad de Dios no es un hecho novedoso porque Dios ya ha sido virtualizado en las Sagradas Escrituras ${ }^{48}$; la virtualización se refiere al proceso que va de la experiencia a la plasmación escrita del texto sagrado, donde no se desconoce el influjo cultural, la comprensión simbólica que subyace en experiencia de Dios, las tradiciones que hay en cada uno de los textos, entre otros asuntos propios

\footnotetext{
${ }^{45}$ Francisco, "Mensaje para la XLVIII Jornada Mundial de las Comunicaciones Sociales. 'Comunicación al servicio de una auténtica cultura del encuentro' (2014)".

${ }^{46}$ Spadaro, Ciberteología. Pensar el cristianismo en tiempos de red.

${ }^{47}$ Castells, La era de la información. La sociedad red; Velásquez, La virtualización en el escenario contemporáneo de la educación; Velásquez, "La sociedad de consumo en La Caverna de Saramago. La aplicación de la función purgativa de la obra literaria"; Baudrillard, La sociedad de consumo: sus mitos y sus estructuras. ${ }^{48}$ Spadaro, Ciberteología. Pensar el cristianismo en tiempos de red.
} 
de los estudios bíblicos ${ }^{49}$. Lo novedoso del asunto reside en que ahora se presenta en pantallas interactivas que involucran ejercitar más de un sentido.

Internet ha acaparado todo el sistema comunicacional global de las sociedades desarrolladas o en vías de desarrollo ${ }^{50}$. Millones de personas hacen de su realidad una virtualidad, porque constantemente permanecen en la red ${ }^{51}$. La permanencia en Internet no se debe exclusivamente a que estemos conectados en un laptop sino al solo el hecho de llevar un dispositivo comunicativo en el bolsillo (como un smartphone) con el que estamos virtualmente presentes en la red $^{52}$, disponibles a los contactos, abiertos a una gran caja mágica multimedia que configura la sociedad red ${ }^{33}$.

De ahí que no se puede desconocer el hecho de que ya somos realmente virtuales, virtualmente reales. En otras palabras: somos esencia y apariencia, es decir, esencia en cuanto personas y apariencia por los rasgos que se dejan ver de nuestra personalidad en la red ${ }^{45}$. Si se ha virtualizado la existencia, entonces es necesario comprender la vida misma en relación directa con la red ${ }^{55}$, de tal forma que podemos entender cómo los dinamismos propios del entorno simbólico del ser humano se recrean en la red.

Uno de esos campos que hacen parte del entorno simbólico del ser humano es la experiencia de Dios, la vivencia de la fe, la religiosidad ${ }^{56}$. Internet ofrece gran variedad de propuestas que hacen parte del gran supermercado espiritual de la actualidad ${ }^{57}$. Solo basta con digitar algunas palabras sobre Dios, la fe, la transcendencia -entre otros conceptos teológicos-, para acceder a una lista variada y rica de propuestas al servicio del navegante.

Sin pretender legitimar cuál de esas experiencias es válida, nos parece pertinente resaltar que la dimensión religiosa de la persona tiene su asidero en la red. Le corresponde al internauta discernir una verdadera experiencia de Dios, en el gran

${ }^{49}$ Paul Ricoeur destaca, en Finitud y culpabilidad, los elementos que convergen en la elaboración del texto bíblico, aquellos que se refieren a la mancilla, la culpa y la piedad. En esos textos, en términos de Pierre Levy, se virtualiza la experiencia de pecado (Levy, ¿Qué es lo virtual?).

${ }^{50}$ Castells, La galaxia Internet.

${ }^{51}$ Ibíd.

${ }^{52}$ Spadaro, Ciberteología. Pensar el cristianismo en tiempos de red.

${ }^{33}$ Castells, La galaxia Internet.

${ }^{54}$ Velásquez, "La dimensión temporal del Dasein en la era virtual: para una hermenéutica existencial del tiempo".

${ }^{5}$ Ibíd.

${ }^{56}$ Cassirer, Antropología filosófica: introducción a una filosofía de la cultura.

${ }^{57}$ Fernández, “Aguinaldo 2016. ¡Con Jesús! Recorramos juntos la aventura del Espíritu”. 
movimiento espiritual que se ha gestado en el siglo, de tal forma que pueda, de forma crítica, encontrar oportunidades para el cultivo de la vida espiritual ${ }^{58}$.

En definitiva, es una afirmación teológica de gran envergadura que Dios se hace virtual para que la persona que navega en Internet pueda salir de sí misma, despegar los ojos de la pantalla y ver al otro que participa también en la virtualidad, para de esta forma crear movimientos de solidaridad, transformación social que comunican en la virtualidad humana la Palabra de Dios: Jesús. Del mismo modo como el Señor se ha hecho carne y puso su morada de campaña entre nosotros (Jn 1,14), él se ha hecho virtual para hacer que nuestra mirada sea dirigida al mundo real: escenario privilegiado donde se hace explícita la comunicación de Dios en la historia humana.

\section{El acompañamiento espiritual virtual: alcances y límites}

Esta sección se propone en indagar acerca de las características del acompañamiento espiritual virtual, sus alcances determinados por el grado de vinculación vital y referencial con el entorno virtual de interacción social y sus límites, por la capacidad de tomar distancia del mundo virtual y afectar el mundo real de interacción humana. Se entiende por acompańamiento:

[...la acción de] ayudar a las personas en su proceso de crecimiento en la fe y en orden a clarificar y discernir la voluntad de Dios, y llegar a un compromiso y opción vocacional mediante la Palabra de Dios, los sacramentos y la oración. Habrá que cuidar el compromiso apostólico, la escucha, el diálogo, el testimonio y otras muchas claves, respetando el desarrollo de cada persona que camina hacia la configuración con Cristo. ${ }^{59}$

El acompañamiento espiritual virtual consiste, entonces - de acuerdo con Salvador García- en facilitar los medios necesarios en el escenario digital de interacción humana para el crecimiento en la fe, de forma que la persona que permanece en la red pueda discernir, de igual modo, la voluntad de Dios, y de esta forma proyectarse en el mundo según la opción vocacional a la que ha sido llamada. Esta modalidad de acompañamiento posibilita la resolución progresiva y dinámica de la pregunta por el sentido de la vida y conduce a la persona a liberarse, de igual modo, cuando la red no contribuye a su formación espiritual.

Las tecnologías se convierten, en este sentido, en una herramienta comunicativa que hace posible la interacción entre el acompañante y la persona que busca en la orientación perspectivas posibles de proyecto. Ahora bien, no se trata de una consulta

\footnotetext{
${ }^{58}$ Torralba Roselló, Inteligencia espiritual.

${ }^{59}$ García, El acompañamiento espiritual. Un ministerio de ayuda, 21.
} 
profesional, ni mucho menos de una instancia unidireccional, sino que surge en todo su sentido el diálogo caracterizado por la fusión de horizontes y la ampliación de la visión del mundo, en la que los dialogantes del proceso juegan con los roles de la comunicación. Esto es posible porque en el mundo virtual los roles no son estáticos sino dinámicos ${ }^{60}$.

Las redes sociales, en efecto, constituyen un escenario virtual privilegiado en el mundo juvenil que permite la relación comunicativa entre diversas personas.

El impacto de las redes sociales en la vida de los jóvenes no puede ser subestimada. Las redes sociales son una parte significativa de la identidad y del estilo de vida de los jóvenes. Los ambientes digitales tienen un gran potencial para unir personas distantes geográficamente, como nunca antes. El intercambio de información, ideales, valores, e intereses comunes actualmente es más posible. El acceso a herramientas de aprendizaje online ha abierto oportunidades educativas para jóvenes en zonas remotas y ha traído el mundo del conocimiento al alcance de un clic. ${ }^{61}$

Los entornos multimedia que ofrece Internet, además de permitir la comunicación, disponen de diversas herramientas multimedia en formatos de audio, texto y video, que posibilitan que la relación con el mundo virtual sea interactiva y significativa. El intercambio de información en las redes sociales ayuda a generar espacios de participación juvenil en la que se comparte, desde asuntos banales hasta cuestiones profundas y realmente vitales, relacionadas directamente con el sentido de la vida. Si el mundo es cada vez más virtual, y los jóvenes participan constantemente de esta forma de socialización humana, tiene sentido pensar la necesidad de aprovechar los diversos ambientes virtuales de aprendizaje y comunicación virtual, para ahondar en lo profundo del misterio de lo humano. Así se puede constatar en el documento presinodal:

Muchos factores influyen en la habilidad de los jóvenes para discernir su vocación, entre los cuales se encuentran: la Iglesia, las diferencias culturales, las exigencias del trabajo, el mundo digital, las expectativas de la propia familia, la salud y el bienestar mental, el ruido, la presión de sus compañeros, los escenarios políticos, la sociedad, la tecnología, etc. Muy pocos jóvenes aprovechan las oportunidades que el silencio, la introspección, la oración, la lectura de las Escrituras, y el mayor conocimiento de uno mismo, pueden ofrecerles. Existe la necesidad de una mejor introducción en estas prácticas. Involucrase en grupos

\footnotetext{
${ }^{60}$ Ibíd.

${ }^{61}$ Oficina de Prensa de la Santa Sede, "Documento de la Reunión presinodal para la preparación de la XV Asamblea General Ordinaria del Sínodo de los Obispos 'Los jóvenes, la fe y el discernimiento vocacional' (19-24 de marzo de 2018)".
} 
de fe, en movimientos y en comunidades con intereses comunes, también pueden ayudar a los jóvenes en su discernimiento. ${ }^{62}$

El mundo digital ofrece una variada amalgama de posibilidades para resolver de forma dinámica y progresiva el significado de la existencia y la vocación a la que cada ser humano está llamado a realizar. La determinación existencial del proyecto de vida no tiene una sola mirada; es decir, no se agota en una perspectiva. Hay muchas personas que al valerse de las herramientas comunicativas que ofrece la sociedad en red -como los celulares o laptops- han encontrado consuelo en su vida, han abierto horizontes vitales de sentido y han descubierto el sentido último de su existencia ${ }^{63}$. No hay que escatimar esfuerzos cuando se trata de aprovechar al máximo lo que se puede hacer en el mundo virtual en favor del anuncio del Reino de Dios y la realización auténtica de la humanidad.

Ahora bien, también somos conscientes de los límites del acompañamiento virtual porque, así como se puede hacer de la virtualidad un escenario rico de interacción social, también se puede relegar el más preciado encuentro real-actual entre las personas; se puede correr el riesgo de perder la sensibilidad por el otro, la humanidad de nuestras relaciones interpersonales por atender de manera exclusiva la realidad virtual. Lo advierte el papa Francisco:

El consumismo hedonista puede jugarnos una mala pasada, porque en la obsesión por pasarla bien terminamos excesivamente concentrados en nosotros mismos, en nuestros derechos y en esa desesperación por tener tiempo libre para disfrutar. Será difícil que nos ocupemos y dediquemos energías a dar una mano a los que están mal si no cultivamos una cierta austeridad, si no luchamos contra esa fiebre que nos impone la sociedad de consumo para vendernos cosas, y que termina convirtiéndonos en pobres insatisfechos que quieren tenerlo todo y probarlo todo. También el consumo de información superficial y las formas de comunicación rápida y virtual pueden ser un factor de atontamiento que se lleva todo nuestro tiempo y nos aleja de la carne sufriente de los hermanos. En medio de esta vorágine actual, el Evangelio vuelve a resonar para ofrecernos una vida diferente, más sana y feliz. ${ }^{64}$

Un sano equilibrio entre el mundo real-actual y su representación virtual, que despierte las conciencias de las personas, que facilite la comunicación y la relación, tanto en el mundo virtual como en el real, será el ideal que jalona las determinaciones

\footnotetext{
${ }^{62}$ Ibíd.

${ }^{63}$ Velásquez, "La dimensión temporal del Dasein en la era virtual. Para una hermenéutica existencial del tiempo".

${ }^{64}$ Francisco, "Exhortación apostólica Gaudete et Exsultate sobre el llamado a la santidad en el mundo actual (2018)" 108.
} 
existenciales de cada persona para hacer de este mundo hiperconectado una realidad más humana. Esto es posible si los que hacemos parte del mundo virtual hacemos presencia como verdaderos ciudadanos digitales y ayudamos a que en el mundo virtual -don de Dios fruto del intelecto humano- puedan surgir verdaderos espacios de comunicación y participación.

\section{Alcances}

El impacto social de los medios de comunicación e información es un rasgo evidente de la cultural actual. Tiene sentido pensar, entonces, en los alcances del acompañamiento espiritual virtual, porque allí donde se desenvuelve y permanece la existencia del humano reside la posibilidad de hacer presente el Reino de Dios y conducir una vida justa y humana.

Como ya se ha dicho en la Introducción, los alcances de acompañamiento espiritual virtual están determinados por el grado de vinculación vital y referencial ${ }^{65}$. Se entiende por vínculo vital el lazo estrecho que hay entre dos o más personas; y por vínculo referencial, la constitución de los referentes existenciales que soportan la idealidad del pensamiento utópico ${ }^{66}$.

Los vínculos vitales creados en la red social virtual tienen su soporte en el mundo real de interacción humana, es decir, el mundo de la vida. La generación, creación y fortalecimiento de dicho lazo comunicativo tiene como protagonistas tanto al acompañante como al acompañado; es decir, es una relación entre iguales que buscan -a como dé lugar- dar sentido a la vida en un mundo caótico y superficial. La creación de vínculos vitales supone las siguientes condiciones:

1. Familiaridad: El modo como se gesta la relación entre el acompañante y el acompañado por medio de la red social. Los gestos que la deben caracterizar son la amabilidad, la cercanía, el interés por el otro, el servicio y la entrega. Hay familiaridad cuando es posible la comunicación espontánea con el otro, la cual permite entablar posibles escenarios participativos virtual sobre asuntos vitales como el sentido de la vida.

2. Afecto: Este se entiende por lo menos en dos direcciones: (a) Los afectos que atraviesan la existencia del ser humano y lo determinan como un ser social en relación; por ejemplo, el aprecio por alguien. (b) El conjunto de afecciones que

\footnotetext{
${ }^{65}$ Velásquez, La virtualización en el escenario contemporáneo de la educación.

${ }^{66}$ Velásquez, "Discursos vacíos para oídos estancados: hacia una hermenéutica de la acción”.
} 
logran incidir de manera significativa en el proceso comunicativo; por ejemplo, los vínculos fuertes de socialización humana.

3. Confianza: Un acompañamiento espiritual virtual solo es posible cuando entre las partes del proceso comunicativo hay confianza. Esta se define como la capacidad de apertura, espontaneidad, gozo y alegría que hay en la relación entre el acompañante y acompañado ${ }^{67}$. Por eso tiene sentido pensar que, para que haya verdadera confianza, estén involucrados en algún sentido sentimientos de amistad para y con el otro.

4. Amistad: En las redes sociales comúnmente a los contactos se les denomina "amigos". ¿Pueden llamarse amigos todos contactos que hacen parte de la red social? Quizás solo haya amistad cuando en el proceso ordinario de socialización y comunicación humana ha habido familiaridad, afecto y confianza. La amistad constituye, en este sentido, un presupuesto de mucha importancia para llevar a cabo proceso de acompañamiento virtual.

Por su parte, los vínculos referenciales se caracterizan por las siguientes condiciones:

1. Respeto: La relación entre el acompañado y el acompañante se debe caracterizar por el respeto, es decir, por un alto grado de estima de uno al otro. Se puede constatar que en las redes sociales se gestan relaciones de poco respeto hacia el otro. Si se quieren llevar a cabo procesos de acompańamiento espiritual virtual es necesario el respeto en el proceso comunicativo en cada una de las partes, de forma que en lo virtual se geste un modo concreto de asumir la vida y las relaciones con los demás.

2. Testimonio: La mejor forma de respaldar un acompañamiento espiritual virtual es por medio del testimonio de vida. ¿Cómo se da testimonio en el mundo virtual? Estar en la red, tener virtualizada la existencia supone que no haya una incisión entre la representación virtual y la vida real. Aquí vale la pena resaltar las siguientes afirmaciones: "Haz con los otros seres virtuales como quisieras que traten a ti": O bien: "Actúa de tal manera que tus acciones en lo virtual sean dignas de ser repetidas" 68 .

${ }^{67}$ En La hermenéutica del sujeto, Foucault, al recurrir a la tradición grecorromana de las prácticas del cuidado de sí, resalta la importancia del acompańamiento. Llama la atención, entre estas prácticas, el acompañamiento como forma de cultivar la vida en el espíritu, que tiene como base, según algunos pensadores clásicos, la confianza (Foucault, La hermenéutica del sujeto).

${ }^{68}$ Velásquez, La virtualización en el escenario contemporáneo de la educación. 
3. Ejemplo: Es la presentación de modelos posibles de vida ${ }^{69}$. En el mundo hay numerosos referentes que estimulan el sueño por un mundo mejor. La red Internet permite conocer estos ejemplos de vida, acceder a la información sobre sus luchas, fracasos y esperanzas.

\section{Limites}

Los límites del acompañamiento espiritual virtual están determinados por la capacidad de tomar distancia del mundo virtual, es decir, por la facultad de entablar relaciones no dependientes, ni con la persona (ya sea acompañante o acompañado), ni mediatizado solo por Internet. La comunicación humana, si bien ha ganado muchos escenarios que posibilitan la interacción con el mundo virtual en sus múltiples formas, también se ha convertido en su impedimento más notable. Tomar distancia del mundo virtual se convierte en el límite del acompañamiento espiritual virtual. El "tomar distancia"70 se convierte en una alternativa loable cuando lo virtual es el único modo de socialización y participación humana.

\section{Conclusión}

Las plataformas virtuales de interacción y comunicación son una alternativa viable para direccionar procesos de acompañamiento espiritual, sobre todo, para las personas que han hecho de su virtualidad una realidad.

Los que permanecen en la red son también destinatarios de los procesos evangelizadores de la Iglesia y, por tanto, no se deben escatimar esfuerzos para llevar a cabo dicha modalidad de acompañamiento. Sin embargo, esta forma de direccionamiento existencial no puede reemplazar el encuentro real-actual entre las personas, ni mucho menos agotar las posibilidades que demanda un acompañamiento espiritual. Por tal motivo, a modo de conclusión, proponemos la imagen eclesial hospital de campaña propuesta por el papa Francisco, para entender los alcances y límites de este tipo de acompañamiento.

El papa Francisco propuso una nueva imagen para comprender la misión de la Iglesia en el mundo como un hospital de campaña $a^{71}$. Se refirió a esta imagen para describir el proyecto de la exhortación apostólica Evangelii gaudium, sobre el anuncio del Evangelio en el mundo actual; fue, además, una figura recurrente durante el Año de

\footnotetext{
${ }^{69}$ Velásquez, "Discursos vacíos para oídos estancados: hacia una hermenéutica de la acción”.

${ }^{70}$ Torralba Roselló, Inteligencia espiritual.

${ }^{71}$ Francisco, "Discurso a los participantes en un encuentro organizado por el Consejo Pontificio para la Promoción de la Nueva Evangelización (19 de septiembre de 2014)".
} 
la Misericordia en 2015. ¿Cuáles son las características de un "hospital de campaña”? Según la OMS y la OPS

...un hospital de campaña es 'una infraestructura de atención sanitaria móvil, autocontenida y autosuficiente que se puede desplegar, instalar y expandir, o en su defecto desmantelar, con rapidez para satisfacer las necesidades inmediatas de atención de salud durante un lapso determinado. ${ }^{72}$

Lejos de profundizar minuciosamente en los detalles que componen un hospital de campaña, mencionaremos sus características principales a partir de la anterior definición ${ }^{73}$ :

- $\quad$ Estructura móvil, autocontenida y autosuficiente.

- Se puede desplegar, instalar y expandir.

- Satisface las necesidades inmediatas.

- $\quad$ Tiene un tiempo determinado.

- Se construye en tiempos de crisis.

¿Qué relación hay entre el "acompañamiento espiritual virtual” y la imagen eclesial como hospital de campaña propuesta por el papa Francisco? Como ya se ha resaltado con insistencia, las redes sociales representan una oportunidad para hacer frente a las necesidades de las personas en lo relacionado con el acompańamiento espiritual. Ahora bien, son una oportunidad, mas no la única alternativa.

El acompañamiento espiritual virtual tiene, de alguna manera, las mismas características del hospital de campaña, porque acontece en una estructura móvil como la red Internet, es de fácil acceso, cumple la función de satisfacer necesidades inmediatas -es decir, problemas urgentes por resolver-, tiene un tiempo determinado (no se puede prolongar), y por lo general se acude a esta alternativa en momentos de crisis. Veamos:

1. Internet, una estructura móvil: Las redes sociales son entornos de interacción social virtual flexibles y dinámicos. Se puede permanecer en la red en modo disponible o desactivado, pero siempre presente. El acompañante virtual está a la mano en el dispositivo.

2. Acceso fácil y disponible: Estar conectado a la red por medio de los dispositivos virtuales hace que el acompañante, en cierto modo, esté siempre disponible. Ahora bien, no quiere decir que haya una respuesta inmediata, porque está

\footnotetext{
${ }^{72}$ Organización Panamericana de Salud. Guía de la OMS y la OPS para el uso de hospitales de campaña extranjeros en caso de desastres.

${ }^{73}$ Ibíd.
} 
condicionado a los límites temporales de la existencia humana. De todos modos, el contacto es fácil y siempre disponible.

3. Satisface necesidades inmediatas: Por lo general el acompañamiento espiritual virtual versa sobre asuntos urgentes y de necesaria atención inmediata; por ejemplo, sensación de sinsentido de la vida, intento de suicidio, soledad, angustia, dolor por sus múltiples causas, crisis de fe, entre otros. No obstante, los problemas que necesitan ser atendidos con mayor detenimiento, por lo general no se tratan por medio de los entornos virtuales, sino sirven para concertar citas en las que pueda darse una conversación más detenida y profunda.

4. Tiempo determinado: Debido a que el acompañamiento espiritual virtual atiende necesidades inmediatas, tiene sentido pensar que su duración es limitada y siempre remite a una instancia mayor y profesional, según sea el caso. Por ejemplo, si un joven contacta a un acompañante virtual y plantea los problemas sobre el sinsentido de la vida, se le debe escuchar de forma atenta y luego remitirlo a un especialista, ya sea en el campo de la psicología o de la psiquiatría.

5. Tiempos de crisis: se acude a un acompañamiento espiritual virtual, por la urgencia de la necesidad, debido a su carácter inmediato y disponible. Las crisis que comporta la existencia humana de los jóvenes son cada vez más acentuadas. Por eso es necesario el acompañamiento que involucra de manera activa procesos de direccionamiento que resuelven, de forma paulatina y provisional, el sentido de las cosas y, de esta forma, tener un panorama de las alternativas de solución.

\section{Referencias}

Arboleda, Carlos. "Evangelizar la cibercultura. Los retos de la ciberteología”. Veritas 38 (2017): 163-181.

Baudrillard, Jean. El sistema de objetos. México: Siglo XXI Editores, 2003. . La ilusión vital. Buenos Aires: Siglo XXI Editores, 2002. . La sociedad de consumo: sus mitos y sus estructuras. Madrid: Siglo XXI Editores, 2009.

Bustamante, Orlando. La práctica de la dirección espiritual en la vida y enseñanzas de San Francisco de Sales. Navarra: Universidad de Navarra, 2006.

Cassirer, Ernst. Antropología filosófica: introducción a una filosofía de la cultura. México: Fondo de Cultura Económica, 1967. 
Castells, Manuel. La era de la información. La sociedad red. Vol. 1. México: Siglo XXI Editores, 2006.

. La galaxia Internet. Reflexiones sobre Internet, empresa y sociedad. Traducción de Raúl Quintana. España: Areté, 2001.

Concilio Vaticano II. "Constitución pastoral Gaudium et spes sobre la Iglesia en el mundo actual (1965)". Vatican, http://www.vatican.va/archive/hist councils/ii vatican council/documents/vat-ii const 19651207 gaudium-et-spes sp.html (consultado el 7 de julio de 2019).

- "Decreto conciliar Inter mirifica sobre los medios de comunicación social (1963)". Vatican, http://www.vatican.va/archive/hist councils/ii vatican council/documents/vat-ii_decree_19631204_inter-mirifica_sp.html (consultado el 20 de mayo de 2019).

Fernández, Don Ángel. "Aguinaldo 2016. ¡Con Jesús! Recorramos juntos la aventura del Espíritu". YouTube, https://www.youtube.com/watch?v=1hnxuIWKAlU (consultado el 20 de mayo de 2019).

Foucault, Michel. La hermenéutica del sujeto. Madrid: Akal, 2005.

Francisco. "Discurso a los participantes en un encuentro organizado por el Consejo Pontificio para la Promoción de la Nueva Evangelización (19 de septiembre de 2014)". Vatican, http://www.vatican.va/content/francesco/es/speeches/2014/ september/documents/papa-francesco_20140919_nuova-evangelizzazione. html (consultado el 28 de febrero de 2019).

- "Exhortación apostólica Gaudete et exsultate sobre el llamado a la santidad en el mundo actual (2018)". Vatican, http://www.vatican.va/content/francesco/ es/apost_exhortations/documents/papa-francesco_esortazione-ap_20180319 gaudete-et-exsultate.html (consultado el 19 de marzo de 2019).

- "Mensaje para la XLVIII Jornada Mundial de las Comunicaciones Sociales. 'Comunicación al servicio de una auténtica cultura del encuentro' (2014)”. Vatican, http://www.vatican.va/content/francesco/es/messages/communications/documents/papa-francesco 20140124 messaggio-comunicazioni-sociali. html (consultado el 20 de abril de 2019).

- "Mensaje para la XLIX Jornada Mundial de las Comunicaciones Sociales. 'Comunicar la familia: ambiente privilegiado del encuentro en la gratuidad del amor' (2015)". Vatican, https://www.vatican.va/content/francesco/es/ messages/communications/documents/papa-francesco 20150123 messaggiocomunicazioni-sociali.html (consultado el 27 de marzo de 2019). 
. "Mensaje para la 50 Jornada Mundial de las Comunicaciones Sociales. 'Comunicación y misericordia: un encuentro fecundo' (2016)”. Vatican, https:// www.vatican.va/content/francesco/es/messages/communications/documents/ papa-francesco_20160124_messaggio-comunicazioni-sociali.html (consultado el 15 de mayo de 2019).

. "Mensaje para la 51 Jornada Mundial de las Comunicaciones Sociales. 'No temas, que yo estoy contigo' (Is 43,5). Comunicar esperanza y confianza en nuestros tiempos' (2017)". Vatican, https://www.vatican.va/content/francesco/ es/messages/communications/documents/papa-francesco 20170124 messaggio-comunicazioni-sociali.html (consultado el 26 de julio de 2019).

. "Mensaje para la 52 Jornada Mundial de las Comunicaciones Sociales. 'La verdad os hará libres (Jn 8,32). Fake news y periodismo de paz' (2018)”. Vatican, https:/www.vatican.va/content/francesco/es/messages/communications/ documents/papa-francesco 20180124 messaggio-comunicazioni-sociali.html (consultado el 30 de junio de 2019).

García, Salvador. El acompañamiento espiritual. Un ministerio de ayuda. Bogotá: Paulinas, 2001.

Guitián, Gregorio. "El Vaticano II y los medios de comunicación: a los cincuenta años de Inter mirifica". Scripta Theologica 43 (2011): 621-643.

Heidegger, Martin. La pregunta por la cosa la doctrina kantiana de los principios trascendentales. Chile: Orbis, 1975.

Herranz, Mariano. San Pablo en sus cartas. Madrid: Encuentro, 2008.

Juan Pablo II. “Carta apostólica 'El rápido desarrollo' a los responsables de las comunicaciones sociales (2005)”. Vatican, http://www.vatican.va/content/john-paul-ii/ es/apost letters/2005/documents/hf jp-ii apl 20050124 il-rapido-sviluppo. html (consultado el 20 de mayo de 2019).

Levy, Pierre. ¿Qué es lo virtual? Barcelona: Paidós, 1999.

McLuhan, Marshall. Comprender los medios de comunicación. Las extensiones del ser humano. Barcelona: Paidós, 1996.

- La aldea global. Transformaciones en la vida y los medios de comunicación mundiales en el siglo XXI. Barcelona: Gedisa, 1993.

Oficina de Prensa de la Santa Sede. "Documento de la Reunión presinodal para la preparación de la XV Asamblea General Ordinaria del Sínodo de los Obispos 'Los jóvenes, la fe y el discernimiento vocacional' (19-24 de marzo 
de 2018)". Vatican, https://press.vatican.va/content/salastampa/es/bollettino/ pubblico/2018/03/24/doc.html (consultado el 24 de febrero de 2019).

Organización Panamericana de Salud. Guía de la OMS y la OPS para el uso de hospitales de campaña extranjeros en caso de desastres. Washington, D. C.: OMS/OPS, 2003.

Pablo VI. "Exhortación apostólica Evangelii nuntiandi acerca de la evangelización en el mundo contemporáneo (1975)". Vatican, http://www.vatican.va/content/ paul-vi/es/apost exhortations/documents/hf p-vi exh 19751208 evangeliinuntiandi.html (consultado el 30 de septiembre de 2018).

Pontificia Comisión para los Medios de Comunicación Social. "Instrucción pastoral Communio et progressio sobre los medios de comunicación social (1971)". Vatican, https://www.vatican.va/roman_curia/pontifical_councils/pccs/documents/rc pc pces doc 23051971_communio_sp.html (consultado el 7 de diciembre de 2018).

Pontificio Consejo para las Comunicaciones Sociales. "Criterios de colaboración ecuménica e interreligiosa en las comunicaciones sociales (1989)”. Vatican, http://www.vatican.va/roman_curia/pontifical_councils/pccs/documents/rc pc pccs doc 04101989 criteria sp.html (consultado el 23 de enero de 2019). . "Ética de las comunicaciones sociales (2000)". Vatican, https://www. vatican.va/roman curia/pontifical councils/pccs/documents/rc pc pccs doc 20000530 ethics-communications sp.html (consultado el 30 de julio de 2019).

. "Ética en Internet (2002)". Vatican, https://www.vatican.va/roman curia/ pontifical councils/pccs/documents/rc pc pccs doc 20020228 ethicsinternet sp.html (consultado el 10 de mayo de 2018).

. “Ética en la publicidad (1997)”. Vatican, https://www.vatican.va/roman curia/ pontifical councils/pccs/documents/rc pc pccs doc 22021997 ethics-inad sp.html (consultado el 20 de septiembre de 2018).

- "Instrucción pastoral Aetatis novae sobre las comunicaciones sociales en el vigésimo aniversario de Communio et progressio (1992)". Vatican, https://www. vatican.va/roman curia/pontifical councils/pccs/documents/rc pc pccs doc 22021992 aetatis sp.html (consultado el 14 de marzo de 2019).

. "La Iglesia en Internet (2002)". Vatican, https://www.vatican.va/roman curia/ pontifical councils/pccs/documents/rc pc pccs doc 20020228 churchinternet sp.html (consultado el 23 de agosto de 2018). 
. "Pornografía y violencia en las comunicaciones sociales: una respuesta pastoral (1989)". Vatican, http://www.vatican.va/roman curia/pontifical councils/pccs/ documents/rc_pc_pccs_doc_07051989_pornography_sp.html (consultado el 30 de junio de 2019).

Ricoeur, Paul. Finitud y culpabilidad. Madrid: Trotta, 2011.

Sartori, Giovanni. Homo videns: sociedad teledirigida. Buenos Aires: Taurus, 1998.

Sínodo de los Obispos. "XV Asamblea General Ordinaria "Los jóvenes, la fe y el discernimiento vocacional. Documento final (2018)". Vatican, http://www.vatican. va/roman curia/synod/documents/rc synod doc 20181027 doc-final-instrumentum-xvassemblea-giovani sp.html (consultado el 18 de mayo de 2019).

Spadaro, Antonio. Ciberteología. Pensar el cristianismo en tiempos de red. Barcelona: Herder, 2014.

Torralba Roselló, Francesc. Inteligencia espiritual. Barcelona, Plataforma Editorial, 2010.

Vattimo, Gianni. "La sociedad transparente". En En torno a la posmodernidad, por G. Vattimo y otros, 9-19. Barcelona: Anthropos, 1990.

Velásquez, Édgar. "Discursos vacíos para oídos estancados: hacia una hermenéutica de la acción”. Revista de Filosofía UCSC 17/1 (2018): 91-115.

. "La dimensión temporal del Dasein en la era virtual: para una hermenéutica existencial del tiempo". Versiones 11, $2^{\circ}$ época (2017): 40-68. - "La sociedad de consumo en La Caverna de Saramago. La aplicación de la función purgativa de la obra literaria”. Escritos 51 (2015): 497-524.

. "La virtualización. Aproximación desde Manuel Castells y Jean Baudrillard". Pensamiento humanista. 11 (2014): 31-53.

. La virtualización en el escenario contemporáneo de la educación. Saarbrücken: Académica Española, 2017. 